\title{
Protée
}

\section{Les régimes répétitifs de l'acte de mâcher}

Entre exacerbation et amuissement

\section{Didier Tsala Effa}

Volume 38, numéro 2, automne 2010

Répétition et habitude dans les pratiques quotidiennes

URI : https://id.erudit.org/iderudit/044950ar

DOI : https://doi.org/10.7202/044950ar

Aller au sommaire du numéro

\section{Éditeur(s)}

Département des arts et lettres - Université du Québec à Chicoutimi

ISSN

0300-3523 (imprimé)

1708-2307 (numérique)

Découvrir la revue

Citer cet article

Effa, D. T. (2010). Les régimes répétitifs de l'acte de mâcher : entre exacerbation et amuïssement. Protée, 38(2), 41-48. https://doi.org/10.7202/044950ar
Résumé de l'article

L'article, à partir d'une analyse sémiolinguistique, tente d'établir les règles d'organisation des régimes répétitifs de l'acte de mâcher. Ces régimes varient principalement du fait des formes matérielles par lesquelles ils opèrent. Le fait répétitif de l'acte de mâcher peut être intentionnel, orienté vers un but : on mâche expressément pour avaler, se nourrir. Le régime répétitif ici se justifie par son exacerbation et le contrôle (bien mâcher, mâcher longuement). Le fait répétitif de l'acte de mâcher peut aussi être purement mécanique et réflexe, incontrôlé. Même si, matériellement, il opère naturellement, le régime répétitif de l'acte de mâcher ici est de l'ordre de l'amuïssement. L'acte de mâcher ne se justifie plus par une visée $a$ priori, il devient une simple opération figurative : telle manière de mâcher peut traduire un trait d'ennui, de nervosité ou même rien du tout ; mâcher peut se réduire à un simple acte médiatique qui n'opère que pour cela, etc. 


\section{LES RÉGIMES RÉPÉTITIFS DE L'ACTE DE MÂCHER: ENTRE EXACERBATION ET AMUIISSEMENT}

Didier TSALA EFFA

Cet article participe d'un projet global dont le but est de poser un regard sur le fait répétitif en tant que forme sémiotique et la façon dont il se manifeste dans nos usages et à travers nos pratiques; quelles articulations, quelle structure canonique? Une manière de procéder serait de partir de discours construits, quels qu'ils soient, et de tenter d'y retrouver différents modes d'existence du fait répétitif, les plus manifestes ou les plus spécifiques. Le résultat reviendrait à établir un système du fait répétitif, intrinsèque à ces discours. Une autre manière serait de se risquer à la construction d'une théorie générale, en partant de virtualités existantes pour en élaborer les principes de fonctionnement. Nous avons choisi de procéder par une approche lexico-sémantique, en partant de la définition de base du dictionnaire. Il s'agit pour nous d'une précaution méthodologique, afin d'obtenir de façon ramassée ce qu'il serait fastidieux de construire sur un plus long terme. La lexicalisation n'est pas un phénomène second des configurations. Elle prend appui, effectivement, sur les usages et se construit à partir de choix et d'exemples qu'on observe dans la pratique discursive. Pour une grande part, elle peut donc être considérée comme un condensé des discours construits, faute de pouvoir établir un corpus plus exhaustif.

Pourquoi le verbe mâcher? Notre point de départ est tout aussi économique. De toutes les activités humaines, s'il est un acte qui traduit par sa manifestation même l'idée de répétition, c'est bien l'acte de mâcher; un acte mécanique, souvent pulsionnel, opéré de façon plus ou moins consciente, mais aussi une pratique socialisée. En partant de ces considérations préalables, nous pensons pouvoir isoler un corpus de règles types qui permette d'expliquer, sans doute différemment, de quelle manière l'acte répétitif fonctionne en tant que forme sémiotique. Après avoir exploré la définition du verbe mâcher en termes de configuration lexico-sémantique, nous interrogerons notamment les principes structurants de l'acte de mâcher comme acte mécanique, puis les principes qui le justifient parfois en tant qu'acte maîtrisé, orienté vers un but. Quel éclairage ces principes jettent-ils sur l'élaboration des conditions de description de l'acte répétitif en tant que forme sémiotique? Telle sera notre démarche.

\section{DÉFINITION LEXICALE ET CONFIGURATION}

Le Petit Robert présente le verbe mâcher sous trois segments définitionnels dont nous pouvons retenir les caractérisations suivantes: 
- un acte performantiel, rythmique: "1. Broyer, écraser avec les dents, par le mouvement des mâchoires, avant d'avaler. Mâcher du pain, de la viande $\rightarrow$ mastiquer;

- un mouvement aspectuel: «2. Triturer longuement dans sa bouche (une substance non comestible qu'on rejette). Mâcher du bétel, du tabac ( $\rightarrow$ chiquer). Mâcher du chewing-gum. Spécialt Papier mâché. Fig. $\rightarrow$ Remâcher»;

- une modalité d'effectuation: «3. Couper sans faire un section nette, en déchirant. Lame mal aiguisée qui mâche le bois». (1993: 1318)

\section{MÂCHER COMME EXACERBATION D'UN ACTE RÉPÉTITIF}

Le premier segment projette la perspective d'une opération processuelle. Le verbe mâcher est envisagé comme une phase intermédiaire en vue d'une action complémentaire. En tant que tel, il amorce et accompagne un mouvement orienté vers un but. On suppose un changement d'état qui devrait se justifier en conséquence par la capacité et l'efficience d'une instance opératrice (objet/sujet) à agir sur l'objet de transformation. Il y a quelque chose à transformer et l'acte de mâcher y conduit par ce fait. Il opère ainsi comme un fait de performance.

Toutefois, le détail de la définition attire l'attention sur une particularité syntaxique de cet acte. Alors qu'on s'attendrait à un acte unique ou alors à des actes consécutifs, nettement identifiés, trois séquences de degrés opérationnels différents sont mises à contribution: l'action directe et immédiate des dents sur l'objet à transformer, l'action du mouvement des mâchoires sur les dents et la précédence à l'ingurgitation sur ce mouvement des mâchoires. Or, malgré cette succession, les relations entre ces séquences ne sont pas des relations de consécution simples, comme on en a l'habitude avec les formes de performance processuelle où, pour parvenir à son objet, le sujet est amené à diversifier ses actions, les unes faisant suite aux autres, en les introduisant au fur et à mesure.

Les relations entre ces séquences laissent voir une forme de structuration qui, d'une séquence à l'autre, déploie un jeu interactionnel peu banal. Quatre instances actantielles sont en considération: les dents, le mouvement (des mâchoires), les mâchoires en tant que telles et la précédence de l'ingurgitation.
Les dents d'abord. Alors que, vis-à-vis de l'objet à transformer, les dents apparaissent comme sujet opérateur, s'exerçant directement sur la matière, on observe que cette fonction, par ailleurs, ne leur est associée que du fait exprès du mouvement des mâchoires. Ainsi, les dents se trouvent alors d'un autre côté et quasi rétrogradées à une fonction purement instrumentale. Sujet opérateur vis-à-vis de l'objet à transformer, les dents combinent d'autre part un statut de «sujet délégué».

Ensuite, le mouvement des mâchoires. Le premier constat est leur indéfinition à l'intérieur des séquences. On pourrait imaginer raisonnablement que ce mouvement est le fait d'une intentionnalité qui serait celle d'un mâcheur indiqué. Mais la définition du dictionnaire met l'accent uniquement sur leur effet opératoire, une simple mécanique, dirons-nous. Or, nous avons souligné le statut subjectif de ce mouvement vis-à-vis des dents. À l'image des dents, on observe aussi une dualité du mouvement des mâchoires en tant qu'instance actantielle, à la fois en position subjectale vis-à-vis des dents et en même temps en position de non-sujet, sans intentionnalité.

Quant aux mâchoires elles-mêmes, si on comprend qu'elles servent de support au mouvement qui conduit à l'exécution de l'action des dents sur la matière, à l'inverse de celles-ci, situées en aval du mouvement, sujet opérateur et délégué, elles se situent quant à elles en amont. Leur position actantielle est celle d'un dispositif, c'est-à-dire d'une modalité, qui permet le mouvement (position objectale, instrumentale) et par lequel l'existence même du mouvement est possible en tant qu'effet opératoire (position subjectale).

Enfin, la précédence de l'ingurgitation. Il ne s'agit pas directement d'une instance actantielle, comme on le voit. La précédence de l'ingurgitation opère comme la condition contractuelle qui encadre les relations actantielles à l'intérieur des quatre séquences. C'est elle qui, par ses contraintes, leur donne sens. Or, cette précédence se présente structurellement comme une condition suspensive. En somme, loin de préciser un objet effectif et butoir pour l'acte de mâcher et les différentes actions qui s'y rattachent, elle les maintient 
à une stricte phase intermédiaire, pour autre chose qui devra commencer ultérieurement. En tant que telle, nous pouvons en tirer de façon canonique une première forme sémiotique de l'acte de mâcher.

Suite aux jeux interactionnels observés, avec cet encadrement contractuel, c'est clairement de la construction d'une instrumentalisation qu'il est question. Pour chaque séquence, nous avons à l'œuvre deux principes interactionnels, un principe de «subjectalisation" et un principe d' "objectalisation». L'interaction entre les instances actantielles tend à créer une relation de subordination du premier sur le deuxième. Les dents en position de sujet opérateur sont subordonnées au mouvement des mâchoires; le mouvement des mâchoires n'est pas observé dans une intentionnalité, mais uniquement comme un effet opératoire; les mâchoires apparaissent comme des dispositifs alors qu'elles servent de support au mouvement; pour finir, le cadre contractuel se présente comme une suspension narrative.

L'instrumentalisation ainsi définie réside dans cette dualité actantielle. Dans le jeu interactionnel, chaque actant, pourtant projeté dans une dynamique subjectale, n'opère en réalité qu'en tant qu'objet.

La description que les physiologistes donnent de la mastication, synonyme direct de ce premier segment définitionnel, est digne d'intérêt pour permettre de cerner le mieux possible cette dualité:

Première étape de la digestion, la mastication est le résultat des mouvements $d u$ maxillaire inférieur (mâchoire inférieure), seul os du visage à avoir une mobilité.

Les mouvements des maxillaires sont essentiellement le résultat de l'action de deux muscles puissants, les masséters, dont la contraction, en prenant appui sur les os du crâne, mobilise de haut en bas, la mâchoire inférieure et des muscles temporaux.

(Vulgaris médical; texte en ligne) ${ }^{1}$

Pour une autre définition, comparative celle-ci, nous avons ce constat:

Locomotion, mastication et respiration [...]. Ce sont des activités rythmiques semi-automatiques. Une fois le geste enclenché volontairement, sa répétition ne dépend plus de la volonté; lorsqu'on marche, qu'on mastique ou qu'on respire, nous n'avons pas besoin de penser aux gestes à faire.

(UdeMNouvelles; texte en ligne) ${ }^{2}$
Par ces définitions, on le voit, la dualité actantielle se résout en effet dans un acte imperfectif: le déclenchement. La part subjectale des actants se révèle comme une phase intermédiaire pour laquelle il n'est pas attendu que les actants contrôlent leur action. A contrario, un actant ne devient actif que dans la mesure où il se spécialise du fait d'un strict processus causal. Des actions s'exécutent, avec des effets avérés. En revanche, ces actions ne sont en réalité que le fait de conversion, dont l'aboutissement est de transformer en exécution une action entreprise nécessairement par un autre actant.

Néanmoins, il n'est pas réellement question de «mécanismes» (Bordron, 2006) au sens strict de simple transformation d'énergie. Bien qu'envisagés d'un point de vue objectal, les dents, le mouvement des mâchoires, les mâchoires, du fait de leur dualité actantielle, continuent de garder tout de même la possibilité de modifier d'eux-mêmes leur fonctionnement en activant, cette fois, leur subjectalité. Par exemple, une dent réagira d'ellemême à une sensation de froid (parce qu'elle dispose d'un nerf); une mâchoire forcera plus ou moins le mouvement en fonction de la densité ou de la dureté de ce qu'il y a à mâcher, etc. La caractérisation de semi-automatique est explicite à cet effet.

Pour autant, un point particulier mérite d'être précisé quant au déclenchement ainsi posé et l'exécution qu'il induit. "Avant d'avaler", nous dit la définition, avec en complément la précision suivante, sous la forme de deux exemples, "Mâcher du pain, de la viande». Ces deux exemples soulignent des substances dont la matérialité est mise à rude épreuve par la mastication. Ce sont ces substances qui sont l'objet de la transformation. Il faudrait s'y appuyer afin de préciser les premières conditions pour une approche du fait répétitif définitoire de l'acte de mâcher.

$\mathrm{Vu}$ sous l'angle de la mastication, en effet, "Avant d'avaler" pose l'acte de mastiquer comme une stricte modalité narrative, en vue d'un programme ultérieur. La matérialité de la substance devient centrale ainsi, dans la mesure où elle se présente comme la condition évaluative de l'opérationnalité de l'acte de mâcher. En tant que matérialité, elle peut opposer une plus ou moins grande résistance à la mastication, ou alors 
elle peut nécessiter une mastication plus ou moins longue, etc. Ce processus et les règles qui l'articulent projettent la première forme d'expression de l'acte de mâcher en tant qu'acte répétitif.

Nous partirons à dessein de l'imaginaire habituel lié à la mastication et qui organise, dans leurs définitions mêmes, les discours idéologiques de la nutrition, allant des discours strictement physiologistes aux discours les plus hygiénistes.

Une référence vient immédiatement à l'esprit, Horace Fletcher à travers l'ensemble des principes cardinaux qu'il postula et des règles qu'il énonça en faveur d'une mastication souvent à l'extrême (certains discours de Fletcher allaient jusqu'à proposer de mâcher l'eau avant de l'avaler).

Horace Fletcher (1849-1919) fut un fervent défenseur des nourritures «saines» sous l'ère victorienne. Il était surnommé "Le Grand Masticateur» en arguant du fait que la nourriture devrait être mâchée trente-deux fois (soit autant qu'il y a de dents chez un humain) - ou environ 100 fois par minute avant d'être avalée. (H. Fletcher; texte en ligne) ${ }^{3}$

Les principes et l'énoncé de Fletcher mettent l'accent sur deux dimensions notables: une dimension quantitative, la mastication comme performance mesurable, et une dimension qualitative qui se justifie du fait du degré de variation attendu vis-à-vis de la matérialité de la substance à mâcher.

La dimension quantitative confère à l'acte de mâcher une échelle de rapports. En d'autres termes, si l'on doit parler de répétition à propos de tel acte de mâcher, ce dernier trouve sa portée d'abord dans la mesure où il se rapporte à une échelle identifiable, si possible figurativisable. L'échelle retenue dans l'énoncé, trente-deux fois, est bien déterminée et, de ce fait, elle s'appuie effectivement sur une évaluation précise. Mais Fletcher est un idéologue. Par analogie, on observe que trente-deux c'est précisément le nombre de dents dont dispose tout être humain adulte. Justement, tout se passe comme si chaque parcelle de nourriture devait être mâchée une fois par chaque dent pour une mastication conforme. Mais on comprend bien que d'autres formes d'échelle restent possibles, par exemple 100 fois par minute, précise aussi Fletcher. Nous pourrons retenir cette contrainte d'échelle comme un premier principe expressif du fait répétitif définitoire de l'acte de mâcher: un principe quantitatif dont l'horizon signifiant est une échelle identifiable, si possible figurativisable. Ainsi, "Mâcher», au sens de mastiquer, pris dans sa dimension quantitative, ne se justifie pas comme une simple production itérative de purs mouvements mécaniques. Ces mouvements se justifient dans la mesure où, par leur nombre, ils se rapportent à une échelle identifiable, une figure: la répétition par le mâchage n'est pas l'équivalent d'une mécanique de mouvements, elle est un rapport d'échelle.

La dimension qualitative est formulée principalement en termes résultatifs, d'une part sous forme de sanction cognitive, avec un énoncé qui parle clairement de punition: "Il clama que "la Nature châtiera ceux qui ne mastiquent pas" et inventa de multiples justifications raffinées pour cette déclaration" (ibid.); d'autre part sous forme de résultats performantiels: «Son principe est que tous les aliments doivent être délibérément mâchés et non avalés jusqu'à ce qu'ils deviennent liquide» ${ }^{4}$. Ce qui est en jeu, c'est la forme même de la matérialité de la substance. Mâcher, au sens de mastiquer, est ainsi un fait délibératoire: le temps de parvenir à autre chose, de tabler sur la nature de la substance à mâcher, en attente de quelque chose d'autre. C'est ce fait délibératoire qui donne sens à la répétition des mouvements de mastication: il faut mastiquer, mais il faut mastiquer jusqu'à un point contraint. Une lecture immédiate conduirait naturellement à considérer ce point contraint comme une modulation qualitative de l'échelle des rapports posée par la dimension quantitative. Évidemment, la configuration ne suppose pas une relation aussi directe. En revanche, cette configuration confère au mâchage les propriétés d'un processus tensif. Mâcher serait ainsi: effectuer des mouvements répétés jusqu'à un certain terme, critique. Pour l'énoncé de Fletcher notamment, ce terme correspondrait à un degré précis de la transformation de la forme de la substance: «jusqu'à ce qu'ils deviennent liquide». On pourrait articuler ici un deuxième principe définitoire du fait répétitif de l'acte de mâcher. Ce fait apparaît comme un fait délibératoire décrit par des propriétés critiques (tensives?). 
MÂCHER COMME AMUÏSSEMENT 5

D'UN ACTE RÉPÉTITIF

Poursuivons notre exploration, cette fois-ci en considérant le deuxième segment de la définition: «2. Triturer longuement dans sa bouche (une substance non comestible qu'on rejette). Mâcher du bétel, du tabac ( $\rightarrow$ chiquer). Mâcher du chewing-gum. Spécialt Papier mâché. Fig. $\rightarrow$ Remâcher».

La première différence qu'introduit ce deuxième segment définitionnel est actantielle. Elle relève de la forme structurale du verbe «triturer» qui met en relation directement d'un côté le mâcheur, envisagé du point de vue de sa performance, de l'autre côté, l'objet qu'il soumet à son action. Face au premier segment et de ce point de vue, cette différence souligne surtout une variation du principe interactionnel. Causal pour le premier segment définitionnel, ce principe devient ici transitif. C'est la seule action du mâcheur qui est en question, alors que, pour le premier segment, cette action valait surtout par la particularité des relations consécutives qui liaient les dispositifs en cause dans l'acte physiologique de mâcher.

La deuxième différence, qui est en effet une amplification de la première, porte sur la nature de la substance à mâcher (une substance non comestible qu'on rejette). Cette particularité restructure l'acte de mâcher en le destinant à un programme inverse de celui du premier segment. Concomitamment, la définition spécifie cette inversion en donnant l'impression de la dégager de toute transformation de la matérialité de la substance à mâcher. Elle ne semble retenir alors que le fait mécanique du mâchage, "triturer longuement", à l'endroit où le premier segment contraignait le mâchage à un point de transformation identifié, figurativisable, par exemple la transformation de la substance en liquide.

En effet, de la formulation «triturer longuement», nous pouvons dégager d'entrée une propriété aspectuelle, d'une part du fait de l'adverbe «longuement» qui infère un aspect duratif, mais aussi du fait du sémantisme même du verbe triturer qui, par lui-même, induit déjà le même type d'effet aspectuel.

Mais ce sémantisme est encore bien spécifique de l'acte de mâcher dans la mesure où, à cette propriété aspectuelle, il en adjoint une deuxième, fondamentale à ce segment définitionnel: l'usure. Étymologiquement, triturer c'est «broyer en frottant». Or, comme on suppose ici nécessairement une matérialité, cette matérialité ne vaut alors que dans la mesure où elle est éprouvée par une action mécanique. Il en résulte une nouvelle propriété de l'acte de mâcher.

L'acte répétitif supposé par le verbe triturer, en tant que forme d'expression de l'usure, est un processus de transformation qui accorde la priorité non plus directement au sort réservé à la matérialité de la substance, mais à la durée et la progressivité de cette transformation. La définition retient pour exemples "Mâcher du bétel, du tabac ( $\rightarrow$ chiquer). Mâcher $d u$ chewing-gum. Spécialt Papier mâché. Fig. $\rightarrow$ Remâcher».

Pour le premier exemple (auquel nous assimilerons aussi le troisième), divers textes anthropologiques relatant l'usage du bétel ne retiennent à dessein que cette progressivité. Il en est ainsi de cette étude de Bonifacy à propos du laquage des dents en noir chez les Annamites (1907: 437-440). L'étude souligne les vertus conservatrices du bétel, pour préserver la noirceur des dents, définie comme une pratique d'élégance: «L'action de fumer et de chiquer le bétel, disent les Annamites, contribue à conserver la teinte noire des dents» (ibid.: 440). L'acte de mâcher opère comme un effet dilatoire qui permet de mettre entre parenthèses une transformation attendue.

Cet effet dilatoire est courant dans diverses scènes de films westerns à l'époque du mouvement dit "Western spaghetti», où le fait de mâcher ou de chiquer a été utilisé dans bien des scènes pour figurer différents états, des attitudes, notamment lors de scènes d'affrontement: on mâche intensément pour faire impression, pour valoriser la force de sa mâchoire. Pour ne citer que l'un des plus célèbres de ces films, revenons au film Le Bon, la brute et le truand, de Sergio Leone, sorti en 1966, et qui a pour cadre la guerre de Sécession. Trois protagonistes sont en scène, à la recherche d'un coffre de 200000 dollars en pièces d'or volées à l'armée sudiste. On se souvient que Blondin, incarné par Clint Eastwood, tire toujours d'affaire Tuco, coupable de ses propres égarements ou de sa naïveté. Fait notable, Blondin 
ponctue chacune de ses interventions salvatrices par un geste où il chique nerveusement le tabac de son cigare. Concomitamment, ce geste marque toujours la fin de la situation critique dont il sort Tuco, et permet chaque fois de faire un pas de plus vers l'accès au coffre.

Dans la structure du film, le geste de la chique apparaît ainsi, pour chaque scène, comme la deuxième boucle d'une parenthèse, clôturant les principales péripéties qui accompagnent la quête des trois hommes et surtout le maintien du couple Blondin/ Tuco, véritable détenteur des codes du secret du coffre. Le fait répétitif ici est spécialement construit en extension, distribué tout au long du film, ce qui le pondère fortement.

Venons-en aux chewing-gums, deuxième exemple du segment définitionnel. Difficile de tirer quelques conclusions a priori en présupposant uniquement des façons de mâcher, telles que nous les imaginons ou telles que nous pouvons les pratiquer individuellement. En revanche, la manière dont les discours marketing exploitent aujourd'hui en communication la mise en scène et la valorisation des chewing-gums est digne d'intérêt. À l'origine simple gomme à mâcher, le chewing-gum, du fait de multiples innovations commerciales et de marketing, a pris des formes diverses en fonction de différentes attentes supposées des consommateurs. Lors d'une étude en entreprise, l'analyse des discours émis, publicitaires mais surtout sur le packaging, nous a permis d'observer, parmi un nombre important de marques, divers partis pris communicationnels. Progressivement, nous en avons dégagé un système dynamique qui a conduit à poser les conditions d'un micro-univers sémantique des façons de mâcher.

Selon les discours émis, certains chewing-gums sont construits en communication, comme destinés à être mâchés autant que possible, sans arrêt. Il faut les mâcher juste pour les mâcher. En général, on sait quand on les met à la bouche, mais on ne sait plus souvent ni comment, ni quand, ni où, s'en défaire. Maintes fois, on oublie même qu'on les mâche. C'est un peu le "chewing-gum du ruminant». Il est habituel d'observer cette façon de mâcher chez de nombreux sportifs qui y recourent juste avant la compétition ou lorsqu'ils sont en plein effort. Le verbe "triturer", d'un certain point de vue, définit très précisément cette façon de mâcher, lorsqu'il met l'accent sur le fait d'usure qui en résulte. Nous l'avons observé préalablement, s'agissant de chewing-gum, l'usure n'est jamais celle de la substance de la matière, qui ne bouge pas. Cette usure est principalement celle de la conscience du temps: plus on mâche, plus on oublie qu'on mâche et plus on continue de mâcher, comme si le temps s'altérait et disparaissait par la durée de la répétition. L'acte répétitif qui y fait référence est alors un acte indéfini, induisant de ce fait un principe de mâchage qui serait de l'ordre de l'étendue, mâcher encore et encore. Évidemment, il s'agit également ici de la manifestation d'une parenthèse, mais considérée cette fois, non plus du point de vue de la clôture comme précédemment, mais du point de vue de son effectuation dans la durée. Un tel principe de mâchage opère à bien des égards comme un dévoiement. Il ne se justifie pas pour une action même s'il s'y greffe: mâcher pendant une performance sportive, pendant un travail harassant, etc. L'acte du mâchage échappe à toute maîtrise, à tout horizon. Et dans le même temps, le fait répétitif perd de sa teneur. C'est le premier terme de notre micro-univers sémantique.

Face à cette forme de communication et en opposition, d'autres chewing-gums apparaissent sur le mode de l'éclat. Le chewing-gum n'est plus mis en communication dans la perspective d'être trituré, mais parce qu'il transporte autre chose, en tant que média. Nous sommes à l'inverse de l'effet dilatoire induit par l'effectuation de la parenthèse. Cette configuration correspond notamment aux chewinggums composés avec des "fourrés" gourmands et destinés à être mangés (comme des bonbons) plutôt qu'à être simplement mâchés. Le mâchage devient accessoire et purement fonctionnel, pour permettre ponctuellement d'ouvrir quelque chose. Il s'épuise par lui-même dans la mesure où il n'opère que comme un point de transfert. Autrement dit, l'acte répétitif du mâchage peut avoir lieu, simplement, il n'affecte pas en soi la valeur de la transformation programmée, c'est une simple opération pragmatique. Un coup de dent pourrait suffire en guise de mâchage, juste pour 
permettre au chewing-gum de livrer ce qu'il renferme. C'est le mâchage gourmand pour lequel la gomme perd toute consistance. Une fois le transfert du «fourré» effectué, si l'on continue de mâcher, c'est dans une autre perspective, en général, on retombe dans la forme de mâchage précédent, de l'ordre de l'étendue et du dévoiement.

La manifestation du micro-univers sémantique identifié révèle deux autres configurations intermédiaires: une configuration regroupant des chewing-gums dont les communications opèrent sous un mode qui serait de l'ordre de la captation et une configuration avec des communications sous l'ordre de l'entraînement.

La première configuration renvoie aux chewinggums proposés avec pour but de provoquer des effets spécifiques sur le corps, apporter une sensation de froid ou alléger la respiration. On attend du coup de dent qu'il déclenche un effet ciblé. Un seul mouvement de mâchoire ou quelques-uns peuvent suffire. Mais ici aussi, comme dans l'acte précédent, bien que nécessaire, cet acte n'est pas central. Il suffit que le chewing-gum ne procure plus rien, qu'il n'y ait plus d'attente, pour que le mâchage ne soit plus nécessaire. L'acte répétitif associé opère alors strictement de manière opportuniste. Plutôt que de renvoyer au mâchage, il se présente d'abord comme la traduction de configurations discursives ad hoc.

La configuration associée au mâchage par entraînement correspond principalement aux chewinggums supposant cette forme particulière qui consiste à mâcher ostensiblement la gomme avec des effets de percussion marqués par des gestes buccaux expressifs et, si besoin, en faisant du bruit. En général, ces chewing-gums sont caractérisés par une teneur en gomme plus importante que celle des autres types de chewing-gums. Le mâchage est envisagé comme déjà débordé par une première transformation. Avant même d'être mâché, le chewing-gum est déjà une masse profuse, consistante. On le conquiert plus qu'on ne le mâche véritablement. L'acte répétitif afférent, s'il opère néanmoins, est virtualisé en réalité. Ce qui compte, en lieu et place du contrôle de la masse diffuse, c'est en premier la performance "esthétique», faire du bruit, marquer les effets de percussion. La représentation ci-après nous permet de formaliser le micro-univers sémantique qui définit les principes de mâchage du chewing-gum et les effets répétitifs afférents.

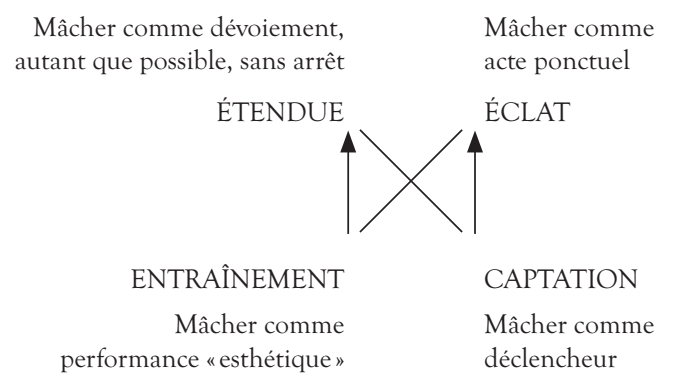

On en vient à la portée structurante de ce deuxième segment définitionnel. Dégagé a priori de toute contrainte vis-à-vis de la matérialité de la substance à mâcher, l'acte de mâcher, réduit à lui-même, conduit à l'amuïssement du fait répétitif qui le sous-tend. À chaque fois et quelle que soit la configuration, il est dévoyé par divers procédés qui tendent à le virtualiser ou à le rendre insignifiant d'une manière ou d'une autre, dans l'attente ou pour autre chose.

Le troisième et dernier segment définitionnel est clairement dénué d'intentionnalité. Il inscrit l'acte de mâcher non dans une perspective de transformation comme les deux premiers, mais dans une perspective purement instrumentale: «3. Couper sans faire une section nette, en déchirant. Lame mal aiguisée qui mâche le bois». Par ce fait, ce segment n'est pas directement concerné par notre problématique, même si on comprend qu'il met en jeu, lui aussi, la matérialité de la substance mâchée. Il est restreint à une description modale, qui ne met pas en scène directement d'organisation répétitive.

\section{CONCLUSION}

Notre objectif était d'interroger, à partir d'une analyse lexico-sémantique du verbe mâcher, la possibilité de quelques conditions de l'acte répétitif dans un de ses usages particuliers. Deux régimes actantiels sont à l'œuvre: un régime causal et un régime transitif.

Le régime causal conduit à une exacerbation du fait répétitif. L'acte de mâcher est envisagé à travers 
un système d'interactions duales qui combinent, pour les différents dispositifs physiologiques constitutifs du mâchage, une opération de subjectalisation et une opération d'objectalisation. Il s'ensuit un phénomène d'instrumentalisation: en même temps qu'on établit la dynamique subjectale d'un dispositif (dent, mouvement des mâchoires, mâchoires, ingurgitation), ce dernier n'est opérant que dans la mesure où il est rétrogradé au statut d'objet modal ou de sujet délégué. Les faits répétitifs qui en résultent relèvent de cette rétrogradation. Ils mettent en scène d'abord des effets de performance. Dans leur effectuation, ces faits se déploient, d'une part, sous la forme d'un rapport d'échelle au sein d'un processus de transformation qui met en rapport la substance d'un objet et la force nécessaire requise pour sa transformation. Ces faits se déploient, ensuite, de façon qualitative. Ils ont en perspective une visée sous la forme d'une attente contrainte, visée qui seule est susceptible de les justifier et de leur donner une valeur.

Le régime actantiel transitif a pour conséquence un amuïssement du fait répétitif. L'acte de mâcher n'est plus envisagé par son effectuation, ni quantitative, ni qualitative. Il ne prend sens que dans la mesure où il s'insère dans une routine qu'il ponctue ou accompagne. Le fait répétitif se déploie alors comme un fait d'usure, non pas l'usure de la matérialité d'une substance, mais celle susceptible d'encadrer la durée et la progressivité d'une transformation. Pour cette usure, nous avons retenu la parenthèse comme une figure type, à travers l'ensemble de ses manifestations, du point de vue de son enclenchement, de sa fermeture, de son effectuation durative. Au final, s'il arrive très souvent qu'on mâche pour quelque chose, on finit par comprendre aussi pourquoi il arrive qu'on mâche sans s'en apercevoir, pour rien.

\section{NOTES}

1. En ligne: http://www.vulgaris-medical.com/encyclopedie/ mastication-7611/physiologie.html (page consultée le 10 mai 2010). 2. En ligne: http://www.nouvelles.umontreal.ca/recherche/sciencesde-la-sante/difficile-de-courir-et-de-macher-de-la-gomme.html (page consultée le 10 mai 2010).

3. En ligne : http://www.regimesmaigrir.com/regimes/fletcher.php (page consultée le 10 mai 2010).

4. En ligne: http://www.ncbi.nlm.nih.gov/pubmed/9693596 (page consultée le 10 mai 2010; notre traduction).

5. Nous envisageons le concept d'amuïssement dans la même perspective que les phonéticiens, qui le définissent comme: «Disparition de la prononciation d'un phonème dans l'évolution phonétique d'une langue (en français, le e muet). En ligne: http://www. universalis.fr/encyclopedie/amuissement/ (page consultée le $10 \mathrm{mai}$ 2010).

\section{RÉFÉRENCES BIBLIOGRAPHIQUES}

BONIFACY [1907]: «Le laquage des Dents en noir chez les Annamites», Bulletins et Mémoires de la Société d'anthropologie de Paris, vol. 8, nº 1 , 437-440.

BORDRON, J.-F. [2006] : "Vers une sémiotique des machines", Nouveaux Actes Sémiotiques, $n^{\circ}$ 109, 3-24.

Greimas, A. J. [1983]: Du Sens II, Paris, Seuil.

Greimas, A. J. et J. FonTANille [1991]: Sémiotique des passions. Des états de choses aux états d'âme, Paris, Seuil.

LÉVI-Strauss, C. [1964]: Le Cru et le Cuit, Paris, Plon. QUÉRÉ, H. [1990] : «Rêveries d'un boudeur solitaire», Cruzeiro semiotico. Semiotica das paixaos, Porto, juillet/janvier, no 11-12, 53-59. LANDOWSKI, E. [2009]: "Avoir prise, donner prise", Nouveaux Actes Sémiotiques. En ligne: http://revues.unilim.fr/nas/document. php?id=2812 (page consultée le 10 mai 2010).

Rey, A. et J. Rey-Debove [1993]: Le Nouveau Petit Robert, Paris, Dictionnaires Le Robert. 